\title{
Aprendizaje y memoria verbal: Comparación entre pacientes con trastorno por consumo de alcohol y trastorno de depresión mayor
}

\section{Learning and verbal memory: A comparison between patients with alcohol use disorder and major depressive disorder}

\author{
Rocío Villa*,**,***, Julia Rodríguez-Revuelta*******, Ashian EsPandian****, Isabel Menéndez- \\ Miranda***, Francesco Dal Santo*,*****, María Paz García-Portilla*******,*****, Gerardo \\ Flórez $* * * * *, * * * * * *$, Julio Bobes*,**,***,*****, Pilar A. SÁiz*,**,***,*****. \\ * Servicio de Salud del Principado de Asturias (SESPA), España. \\ ** Instituto de Investigación Sanitaria del Principado de Asturias (ISPA), España. \\ *** Área de Psiquiatría, Universidad de Oviedo, España. \\ **** Servicio de Psiquiatría del Hospital del Bierzo. Servicio de Salud Mental de Castilla y León (SACYL), España. \\ ***** Centro de Investigación Biomédica en Red de Salud Mental (CIBERSAM), España. \\ ****** Unidad de Conductas Adictivas, Complejo Hospitalario Universitario de Ourense, España.
}

\section{Resumen}

El daño cerebral relacionado con el consumo de alcohol se asocia a alteraciones de las funciones cognitivas, entre las que destacan memoria y aprendizaje verbal. El objetivo principal es evaluar memoria y aprendizaje verbal en una muestra de 111 pacientes con trastorno por consumo de alcohol (TCA) versus 78 con trastorno de depresión mayor (TDM) y 100 controles sanos. La evaluación incluyó variables sociodemográficas y clínicas, la Escala de Hamilton para la Depresión (HDRS) y el Test de Aprendizaje Verbal de California (CVLT). Se utilizó ANOVA de un factor para comparaciones entre los 3 grupos y ANCOVAS bidireccionales incluyendo diferentes covariables. El ANOVA de un factor muestra que los pacientes con TCA y TDM obtienen puntuaciones similares entre sí e inferiores a las del grupo control ( $\mathrm{p}<0,001)$, con excepción del CVLT Guiado (peores puntuaciones en TDM vs TCA, $\mathrm{p}<0,001$ ). Tras incluir como covariables la edad, sexo y los años de estudios completados, persisten las diferencias entre los grupos de TCA y TDM frente al grupo control ( $p \leq 0,003)$ en todos los índices con excepción del CVLT Libre Inmediato y del CVLT Guiado (peor rendimiento en TDM vs TCA, $\mathrm{p}=0,022$ y $\mathrm{p}=0,035$, respectivamente). En el segundo ANCOVA, tras controlar por gravedad de la depresión, únicamente se detectan diferencias entre los pacientes con TCA y los controles sanos ( $\mathrm{p} \leq$ 0,007). Los pacientes con TCA presentan una importante alteración en aprendizaje y memoria verbal al compararlos con pacientes con TDM y con personas sanas.

Palabras clave: Aprendizaje verbal; California Verbal Learning Test; memoria verbal; trastorno de depresión mayor; trastorno por consumo de alcohol.

\begin{abstract}
Brain damage related to alcohol consumption is associated with impairments in cognitive functions, among which memory and verbal learning stand out. The main objective is to evaluate memory and verbal learning in a sample of 111 patients with alcohol use disorder (AUD) versus 78 with major depressive disorder (MDD) and 100 healthy controls. The evaluation included sociodemographic and clinical variables, the Hamilton Depression Scale (HDRS) and the California Verbal Learning Test (CVLT). One-way ANOVA was used for comparisons between the 3 groups and two-way ANCOVAS including different covariates. The one-way ANOVA shows that patients with AUD and MDD had scores similar to each other and lower than those of the control group $(\mathrm{p}<0.001)$, with the exception of the Cued CVLT (worse scores in MDD vs AUD, p <0.001). After including age, sex and years of completed studies as covariates, the differences between the AUD and MDD groups persisted compared to the control group $(\mathrm{p} \leq 0.003)$ in all indices except for the Immediate Free CVLT and the Cued CVLT (worse performance in MDD vs AUD, $\mathrm{p}=0.022$ and $\mathrm{p}=0.035$, respectively). In the second ANCOVA, after controlling for depression severity, differences were only detected between AUD patients and healthy controls $(\mathrm{p} \leq 0.007)$. Patients with AUD present a significant impairment in learning and verbal memory when compared with patients with MDD and with healthy people. Key words: Alcohol use disorder; California Verbal Learning Test; major depressive disorder; verbal learning; verbal memory.
\end{abstract}


$\mathrm{L}$ a mayor parte de los estudios realizados en pacientes con trastornos mentales, en general, y con trastorno por consumo de sustancias, en particular, se centran en aspectos psicopatológicos. Sin embargo, existe escasa atención a los déficits cognitivos asociados a tales trastornos a pesar de su importancia y de la repercusión que éstos tienen en la calidad de vida y la integración social y profesional de los pacientes (Millan et al., 2012).

Existe amplia evidencia de que el uso prolongado y excesivo de alcohol puede provocar daño cerebral estructural y funcional (Crowe, Cammisuli y Stranks, 2019; Le Berre, Fama y Sullivan, 2017; Stavro, Pelletier y Potvin, 2013; Toledo-Nunes, Kipp, Reitz y Savage, 2019) que puede generar desde deterioros sutiles hasta deterioros cognitivos más graves y duraderos como demencia (Brust, 2010; Hayes, Demirkol, Ridley, Withall y Draper, 2016; Svanberg y Evans, 2013; Toledo-Nunes et al., 2019). Esta disfunción cerebral asociada al consumo de alcohol tiene como origen dos mecanismos tóxicos que actúan de forma combinada (Moretti, Caruso, Dal Ben, Gazzin y Tiribelli, 2017). Por un lado, el efecto neurotóxico directo del etanol (Stavro et al., 2013; Wollenweber et al., 2014) y, por otro lado, el asociado al déficit de tiamina que da lugar al Síndrome de Wernicke/Korsakoff cuyo síntoma nuclear es un trastorno grave de la memoria (Kuźma, Llewellyn, Langa, Wallace y Lang, 2014; Maharasingam, Macniven y Mason, 2013; Sachdeva, Chandra, Choudhary, Dayal y Anand, 2016). Por otra parte, la creencia del efecto neuroprotector del consumo moderado de alcohol ha sido cuestionada recientemente, con evidencia de daño en bebedores moderados en comparación con los abstemios (Topiwala et al., 2017).

Cabe destacar, que el daño cerebral relacionado con el consumo de alcohol es un problema de salud frecuentemente infradiagnosticado (Sachveda et al., 2016; Hayes et al., 2016), y se asocia a alteraciones en las siguientes funciones cognitivas: atención, velocidad de procesamiento, tareas visuoespaciales, memoria anterógrada, memoria de trabajo y función ejecutiva (fluidez verbal, resistencia a la interferencia, razonamiento abstracto y flexibilidad cognitiva) (Erdozain et al., 2014; Spear, 2018; Stavro et al., 2013; Villa et al., 2021a).

El grado de deterioro no está únicamente influenciado por la cantidad de alcohol, sino también por el patrón de consumo (Florez, Espandian, Villa y Saiz, 2019; Hayes et al., 2016). Siendo destacable que los episodios de consumos masivos pueden provocar una afectación neuronal más intensa a nivel del hipocampo, hipotálamo y cerebelo, que interferiría en la capacidad de aprendizaje y memoria (Ridley, Draper y Withall, 2013).

Por otro lado, la disfunción cognitiva en el trastorno de depresión mayor (TDM) afecta a diferentes dominios, que incluyen entre otros a la función ejecutiva, la memoria verbal y la atención (Bortolato et al., 2016; Fossatti, Co- yette, Ergis y Allilaire, 2002; Marazziti, Consoli, Picchetti, Carlini y Faravelli, 2010; Roca, Vives, López-Navarro, García-Campayo y Gili, 2015). Estudios previos sugieren que el deterioro cognitivo en la TDM puede ser un dominio de síntomas independiente, más que un epifenómeno de síntomas relacionados con el estado de ánimo (Gregory et al., 2020; Rock, Roiser, Riedel y Blackwell, 2014). Tanto en pacientes con depresión unipolar como bipolar se evidencia un deterioro de memoria y aprendizaje verbal que es habitualmente interpretado como un reflejo de la incapacidad para transferir la información del almacenamiento a corto plazo al almacenamiento a largo plazo (Marazzitti et al., 2010).

Datos previos ponen de manifiesto que existe una alteración en los diferentes índices del test de aprendizaje verbal de California (CVLT) en los pacientes con trastorno por consumo de alcohol (TCA) (Ros-Cucurull et al., 2018; Van Geldorp, Bergman, Robertson, Wester y Kessels, 2012; Villa et al., 2021a; Villa et al., 2021b; Wester, Roelofs, Egger y Kessels, 2014). Si bien, hay estudios previos que analizan la función cognitiva en comorbilidad alcohol y depresión (Hunt, Baker, Michie, y Kavanagh, 2009; Hunt, Kay-Lambkin, Baker y Michie, 2015; Lee et al., 2015), hasta la fecha no existen estudios que comparen la afectación en memoria y aprendizaje verbal en pacientes con TCA versus pacientes con TDM y controles sanos, lo que constituye la principal innovación de este trabajo.

El objetivo principal del presente estudio es evaluar la memoria y el aprendizaje verbal en una muestra de pacientes con TCA en comparación con pacientes con TDM y controles sanos. Basándonos en la literatura previa, nuestra hipótesis de trabajo es que los pacientes con TCA presentarán mayor grado de deterioro en dichas funciones que el resto de la muestra (TDM y controles sanos).

\section{Material y métodos}

\section{Participantes}

La muestra final está compuesta por 111 pacientes con TCA activo (criterios DSM-5) (American Psychiatric Association, 2013) en el momento del reclutamiento, 78 pacientes con TDM (criterios DSM-5) (American Psychiatric Association, 2013) y 100 controles sanos. Los participantes con TCA fueron reclutados en tres dispositivos sanitarios: el Centro de Salud Mental La Calzada de Gijón, la Unidad de Conductas Adictivas del Servicio de Psiquiatría del Complejo Hospitalario de Ourense y el Instituto de NeuroPsiquiatría y Adicciones, Parc de Salut Mar, Barcelona. Los pacientes con diagnostico TDM fueron reclutados en el Centro de Salud Mental II - La Corredoria de Oviedo.

Los pacientes con TCA cumplían criterios DSM-5 de trastorno por consumo de alcohol moderado o grave, cuantificado a lo largo del último mes como un consumo activo $>60$ gramos de etanol/día en varones y $>40$ gramos 
de etanol/día en mujeres y expresaron un claro deseo de controlar su consumo de alcohol. Entre los criterios de inclusión de los pacientes con TCA cabe destacar no tener historia previa de episodios depresivos y tener una puntuación < 5 en la Escala de Hamilton para la Depresión-17 ítems (HDRS) (Hamilton, 1960). Entre los criterios de inclusión del grupo de pacientes con TDM, cabe destacar una puntuación en la escala HDRS $\geq 15$ puntos. Como principales criterios de exclusión para ambos grupos de pacientes (TCA y TDM) mencionar comorbilidad con otro trastorno psiquiátrico según criterios DSM-5, (excepto trastorno por consumo de tabaco), discapacidad intelectual o cualquier enfermedad física grave.

En el grupo control se incluyeron personas sin historia actual o pasada de trastorno mental y sin antecedentes familiares de TCA, ni TDM. Por otra parte, no debían presentar a lo largo del último mes un consumo de alcohol $>30$ gramos de etanol/día.

Todos los participantes en el estudio fueron mayores de 18 años, aceptaron participar en el estudio y firmaron el correspondiente consentimiento informado. El estudio fue aprobado por los siguientes Comités de Ética de la Investigación: Pontevedra - Vigo - Ourense (2016-313), Principado de Asturias (61/14, 06/17 y 142/19) y Parc de Salut Mar (2017-7221-I) y se desarrolló siguiendo la normativa ética y legal sobre la protección de datos personales y estudios con humanos, cumpliendo las directrices de la Declaración de Helsinki (World Medical Association General Assembly, 2013).

\section{Instrumentos de evaluación}

Para la evaluación de la muestra incluida se utilizó un cuestionario ad-hoc (variables sociodemográficas y clínicas) y la versión española de la escala de HDRS (Hamilton, 1960; validación en castellano por Bobes et al., 2003). Por último, el Test de Aprendizaje Verbal de California (CVLT) (Delis, Kramer, Kaplan y Ober, 1987) se utilizó para evaluar diferentes índices de la memoria verbal como son la codificación, el recuerdo libre, guiado y el reconocimiento presentado de manera oral. Para ello el evaluador lee una lista de 16 sustantivos en voz alta (Lista A), a intervalos de un segundo, en orden fijo, durante cinco ensayos de aprendizaje, con el sumatorio de los aciertos se conforma el CVLT Total de Intentos. A continuación, se presenta una lista de interferencias (Lista B) y se procede a preguntar si recuerda la Lista A (CVLT Libre Inmediato). Una vez trascurridos 20 minutos se pide al sujeto que recuerde tantas palabras como sea posible (CVLT Demorado) y después el evaluador ofrece señales o pistas semánticas (CVLT Guiado). Por último, se presenta un ensayo de reconocimiento en el que los participantes deben discriminar las palabras de la Lista A de los distractores (CVLT Reconocimiento).

\section{Análisis de datos}

Los datos fueron analizados utilizando el paquete estadístico SPSS 24.0 (SPSS, Armonk, NY: IBM Corp.). Los datos se presentan utilizando medias (M) y desviaciones estándar (DE) para las variables numéricas y frecuencias y porcentajes para las variables categóricas.

Se utilizó la prueba de Chi-cuadrado $\left(\chi^{2}\right)$ para las variables categóricas, utilizándose la prueba exacta de Fisher para establecer los grupos estadísticamente diferentes siempre que los grados de libertad ( $\mathrm{gl}$ ) fueron superior a 1 y se utilizó el Análisis de la Varianza (ANOVA) de un factor con corrección post hoc de Bonferroni para hacer la comparación, entre los 3 grupos, de las variables numéricas. Posteriormente se llevaron a cabo dos Análisis de la Covarianza (ANCOVA) bidireccionales. En el primero de los ANCOVAs se consideraron como factores fijos el grupo muestral de pertenencia y el sexo y como covariables aquellas variables que la literatura previa pone de manifiesto que influyen en los resultados de los diferentes índices del CVLT, la edad y los años de estudios completados. En el segundo ANCOVA se añadió como otra covariable más la gravedad de la depresión según la HDRS. El nivel de significación estadística establecido en todos los casos fue $\alpha=$ 0,05 (2 colas).

\section{Resultados}

La muestra está compuesta por un total de 289 personas de las cuales 111 tenían diagnóstico de TCA [edad media $(\mathrm{DE})=49,07(8,41)$; hombres: $\mathrm{n}=87(78,4 \%)]$, 78 TDM [edad media (DE) = 52,28 (10,63); hombres: $n$ $=32(41,0 \%)]$ y 100 controles sanos [edad media $(\mathrm{DE})=$ 48,66 (9,57); hombres: $\mathrm{n}=74(74,0 \%)]$. Las principales características sociodemográficas de la muestra se resumen en la tabla 1. Es destacable, que en el grupo de pacientes con TCA la media de años (DE) en situación de dependencia fue de 15,45 $(9,92)$ y la de UBEs / día (durante el último mes) de 9,61 $(6,43)$. Por otra parte, los pacientes con TDM presentaban una gravedad media (DE) de la depresión medida con la HDRS de 21,03 (4,09), compatible con un cuadro de depresión mayor según los criterios de Bech (1996). Por otra parte, tras la realización del correspondiente análisis en función del sexo, de modo independiente, en cada uno de los grupos estudiados, ha podido comprobarse que no existen diferencias estadísticamente significativas entre hombres y mujeres en las diferentes variables sociodemográficas y clínicas incluidas en el estudio.

Para la comparación entre los tres grupos de estudio de los resultados obtenidos en los diferentes índices del CVLT se realizó en primer lugar un ANOVA exploratorio que puso de manifiesto que los grupos de pacientes con TCA y TDM obtenían puntuaciones similares entre sí y estadísticamente inferiores a las del grupo de controles sanos $(p<0,001)$, con la excepción de las puntuaciones 
Tabla 1. Variables sociodemográficas y clínicas de los grupos de estudio.

\begin{tabular}{|c|c|c|c|c|c|}
\hline & $\begin{array}{l}\text { Alcohol }^{1} \\
(n=111)\end{array}$ & $\begin{array}{c}\text { Depresión }{ }^{2} \\
(n=78)\end{array}$ & $\begin{array}{l}\text { Controles }^{3} \\
(\mathrm{n}=100)\end{array}$ & $X^{2}(g l) / F(g l)$ & $p$ \\
\hline \multicolumn{6}{|l|}{ Sexo [n (\%)] } \\
\hline Hombres & $87(78,4 \%)$ & $32(41,0 \%)$ & $74(74,0 \%)$ & $32,403(2)^{\star}$ & $<0,001(1 \text { y } 3 \neq 2)^{\star \star \star}$ \\
\hline Edad [M (DE)] & $49,07(8,41)$ & $52,28(10,63)$ & $48,66(9,57)$ & $3.749(288)^{\star \star}$ & $0,035(2 \neq 3)^{\star \star \star \star}$ \\
\hline Estado civil [n (\%)] & & & & \multirow{5}{*}{$28,960(6)^{\star}$} & $<0,001$ \\
\hline Soltero & $28(25,2 \%)$ & $2(2,6 \%)$ & $27(27,0 \%)$ & & $<0,001(1 \text { y } 3 \neq 2)^{\star \star * *}$ \\
\hline Casado / pareja & $46(41,4 \%)$ & $52(66,7 \%)$ & $56(56,0 \%)$ & & $\leq 0,039(1 \neq 2 \text { y } 3)^{\star \star \star *}$ \\
\hline Separado / divorciado & $33(29,7 \%)$ & $19(24,4 \%)$ & $15(15,0 \%)$ & & $0,032(1 \neq 3)^{\star \star \star}$ \\
\hline Viudo & $4(3,6 \%)$ & $5(6,4 \%)$ & $2(2,0 \%)$ & & NS \\
\hline Años de estudios finalizados [M (DE)] & $13,30(2,88)$ & $13,32(4,76)$ & $12,76(2,42)$ & $0,865(288)^{\star *}$ & 0,422 \\
\hline HDRS [M (DE)] & $1,97(2,02)$ & $21,03(4,09)$ & $1,22(1,76)$ & $1505,96(288)^{\star \star *}$ & $<0,001(1 \text { y } 3 \neq 2)^{\star \star \star \star *}$ \\
\hline Edad de inicio dependencia [M (DE)] & $33,62(9,15$ & - & - & - & - \\
\hline Años de dependencia [M (DE)] & $15,45(9,92)$ & - & - & - & - \\
\hline UBEs / día (último mes) [M (DE)] & $9,61(6,43)$ & - & - & - & - \\
\hline
\end{tabular}

Nota. ${ }^{\star}$ Chi-cuadrado; ${ }^{* *}$ Análisis de la varianza; ${ }^{* \star *}$ Grupos estadísticamente diferentes tras Prueba Exacta de Fisher; ${ }^{* \star * \star G r u p o s ~ e s t a d i ́ s t i c a m e n t e ~ s i g n i f i c a t i v o s ~ t r a s ~}$ corrección post hoc de Bonferroni; $\mathrm{gl}=$ grados de libertad; $\mathrm{M}=$ media; $\mathrm{DE}=$ desviación estándar; HDRS = Escala de Hamilton para la Depresión; UBE = Unidad de Bebida Estándar; NS = no significativo.

Tabla 2. Medias, medias ajustadas, desviaciones estándar y errores estándar para los diferentes índices del California Verbal Learning Test en los diferentes grupos.

\begin{tabular}{|c|c|c|c|c|c|}
\hline & $\begin{array}{l}\text { Alcohol }^{1} \\
(n=111)\end{array}$ & $\begin{array}{l}\text { Depresión }^{2} \\
(n=78)\end{array}$ & $\begin{array}{c}\text { Controles }^{3} \\
(n=100)\end{array}$ & $F(g l)$ & $p(\star \star \star \star \star)$ \\
\hline CVLT Total Intentos [M (DE)] & $45,76(11,61)$ & $45,56(12,16)$ & $53,20(9,80)$ & $14,826(2,286)^{\star \star \star}$ & $<0,001(1$ y $2 \neq 3)$ \\
\hline CVLT Total Intentos [Majustada (SE)] ${ }^{\star}$ & $47,84(1,19)$ & $45,62(1,21)$ & $54,42(1,19)$ & $14,599(2,281)^{\star \star \star \star}$ & $<0,001(1$ y $2 \neq 3)$ \\
\hline CVLT Total Intentos [Majustada (SE)] ${ }^{\star \star}$ & $46,16(1,64)$ & $50,56(3,52)$ & $52,44(1,78)$ & $7,149(2,280)^{\star \star \star \star}$ & $0,001(1 \neq 3)$ \\
\hline CVLT Libre Inmediato [M (DE)] & $9,77(3,32)$ & $9,08(3,41)$ & $12,31(2,82)$ & $26,838(2,286)^{\star \star \star}$ & $<0,001(1$ y $2 \neq 3)$ \\
\hline CVLT Libre Inmediato [Majustada (SE)] ${ }^{*}$ & $10,40(0,34)$ & $9,09(0,34)$ & $12,70(0,34)$ & $28,451(2,281)^{\star \star \star \star}$ & $\begin{array}{c}<0,001(1 \text { y } 2 \neq 3) \\
0,022(1 \neq 2)\end{array}$ \\
\hline CVLT Libre Inmediato [Majustada (SE)] ${ }^{\star \star}$ & $10,19(0,47)$ & $9,70(1,01)$ & $12,45(0,51)$ & $11,155(2,280)^{\star \star \star \star *}$ & $<0,001(1 \neq 3)$ \\
\hline CVLT Libre Demorado [M (DE)] & $10,32(3,35)$ & $9,56(3,37)$ & $12,98(2,90)$ & $29,394(2,286)^{\star \star \star \star}$ & $<0,001(1$ y $2 \neq 3)$ \\
\hline CVLT Libre Demorado [Majustada (SE)] ${ }^{*}$ & $10,79(0,35)$ & $9,67(0,36)$ & $13,25(0,35)$ & $26,575(2,281)^{\star \star \star \star}$ & $<0,001(1$ y $2 \neq 3)$ \\
\hline CVLT Libre Demorado [Majustada (SE)] $]^{\star \star}$ & $10,39(0,49)$ & $10,87(1,05)$ & $12,78(0,53)$ & $11,341(2,280)^{\star \star \star \star}$ & $<0,001(1 \neq 3)$ \\
\hline CVLT Guiado [M (DE)] & $11,42(2,83)$ & $10,56(2,99)$ & $13,64(2,70)$ & $29,171(2,286)^{\star \star \star}$ & $<0,001 \quad(1 \neq 2 \neq 3)$ \\
\hline CVLT Guiado [M (SE) $]^{\star}$ & $11,75(0,32)$ & $10,64(0,32)$ & $13,87(0,32)$ & $26,911(2,281)^{\star \star \star \star}$ & $\begin{array}{c}<0,001(1 \text { y } 2 \neq 3) \\
0,035(1 \neq 2)\end{array}$ \\
\hline CVLT Guiado [M (SE)] ${ }^{\star \star}$ & $11,62(0,44)$ & $10,99(0,, 94)$ & $13,71(0,47)$ & $11,088(2,280)^{\star \star \star \star \star}$ & $<0,001(1 \neq 3)$ \\
\hline CVLT Reconocimiento [M (DE)] & $14,20(2,12)$ & $13,94(2,37)$ & $15,34(1,08)$ & $14,502(2,286)^{\star \star \star}$ & $<0,001(1$ y $2 \neq 3)$ \\
\hline CVLT Reconocimiento [M (SE)]* & $14,37(0,22)$ & $13,89(0,22)$ & $15,40(0,22)$ & $12,351(2,281)^{\star \star \star \star}$ & $\begin{array}{c}0,003(1 \neq 3) \\
<0,001(2 \neq 3) \\
\end{array}$ \\
\hline CVLT Reconocimiento [M (SE)] ${ }^{\star \star}$ & $13,94(0,30)$ & $15,16(0,64)$ & $14,89(0,32)$ & $5,495(2,280)^{\star \star \star \star}$ & $0,007(1 \neq 3)$ \\
\hline
\end{tabular}

Nota. *Covariables: edad y años de estudios finalizados; **Covariables: edad, años de estudios finalizados y puntuación en la Escala de Hamilton para la Depresión (HDRS); ${ }^{* \star *}$ Análisis de la varianza; ${ }^{* * *}$ Análisis de la covarianza; ${ }^{* * * *}$ Grupos estadísticamente diferentes considerando tras corrección post-hoc de Bonferroni CVLT = California Verbal Learning Test; $\mathrm{M}$ = media; $\mathrm{DE}$ = desviación estándar; gl = grados de libertad; SE = error estándar.

obtenidas en el índice CVLT Guiado en la que todos los grupos fueron diferentes entre sí y los pacientes con TDM fueron los que obtuvieron las puntuación más bajas $(p<$ 0,001 ) (ver tabla 2). Posteriormente, se realizaron dos ANCOVAS consecutivos, de modo que en el primero se consideraron como factores fijos el grupo muestral al que pertenecían y el sexo y como covariables la edad y los años de estudios completados y en el segundo ANCOVA se añadió como nueva covariable la gravedad de la depresión según la HDRS (Tabla 2). Tras la realización del primer ANCOVA puede observarse que persistían las diferencias en las puntuaciones obtenidas entre los grupos de TCA y TDM frente al grupo control $(p \leq 0,003)$ en todos los índices con excepción del CVLT Libre Inmediato y del CVLT Guiado, en la que también existían diferencias entre las puntuaciones obtenidas por pacientes con TCA y TDM ( $p=0,022$ y $p$ 
$=0,035$, respectivamente) (Tabla 2). Cabe señalar que, en todos los índices y en todos los grupos, las mujeres obtuvieron puntuaciones estadísticamente superiores a las de los hombres $(p \leq 0,017)$ (Tabla 3$)$. No se observó en ninguno de los índices una interacción estadísticamente significativa entre sexo y grupo tras el correspondiente control por edad y años de educación completados [CVLT Total Intentos: $\mathrm{F}(2,281)=0,569, p=0,567$, parcial $\eta^{2}=0,004$; CVLT Libre Inmediato: $\mathrm{F}(2,281)=0,252, p=0,777$, parcial $\eta^{2}=$ 0,002; CVLT Libre Demorado: F $(2,281)=0,617, p=0,540$, parcial $\eta^{2}=0,004$; CVLT Guiado: $\mathrm{F}(2,281)=0,224, p=$ 0,800, parcial $\eta^{2}=0,002$; CVLT Reconocimiento: F (2, 281) $=0,467, p=0,628$, parcial $\left.\eta^{2}=0,003\right]$ (Tabla 3 ).

Como ya se ha comentado previamente, en el segundo de los ANCOVAS realizados también se incluyó como covariable la puntuación total en la escala HDRS. En la figura 1, puede observarse como para todos los índices las mujeres obtienen puntuaciones significativamente más elevadas que los hombres, independientemente del grupo al que pertenezcan (CVLT Total Intentos: $p<0,001$; CVLT Libre Inmediato: $p<0,001$; CVLT Libre Demorado: $p=0,001$; CVLT Guiado: $p=0,006$; CVLT Reconocimiento: $p=0,027$ ) (Tabla 3). En la tabla 2 puede observarse como tras controlar por la gravedad de la depresión sólo se detectan diferencias estadísticamente significativas entre el grupo de pacientes con TCA y los controles sanos $(p \leq 0,007)$. Por último, señalar que no existe para ninguno de los índices una interacción estadísticamente significativa entre sexo y grupo tras el correspondiente control por edad, años de educación completados y puntuación en la escala HDRS [CVLT Total Intentos: F $(2,280)=0,702, p=0,497$, parcial $\eta^{2}=0,005$; CVLT Libre Inmediato: $\mathrm{F}(2,280)=0,286, p=$ 0,751, parcial $\eta^{2}=0,002$; CVLT Libre Demorado: F (2, 280) $=0,748, p=0,474$, parcial $\eta^{2}=0,005$; CVLT Guiado: F (2, $280)=0,249, p=0,780$, parcial $\eta^{2}=0,002$; CVLT Reconocimiento: $\mathrm{F}(2,280)=0,384, p=0,682$, parcial $\left.\eta^{2}=0,003\right]$ (Tabla 3).

\section{Discusión}

El objetivo principal de este estudio es comparar, utilizando el CVLT, el rendimiento en la memoria verbal de pacientes con TCA y TDM y controles sanos. Como cabría esperar los pacientes con TCA o TDM presentan un peor rendimiento que los controles sanos en todos los índices evaluados por dicha prueba.

Un primer análisis exploratorio pone de manifiesto que la afectación en el aprendizaje, el recuerdo inmediato y el recuerdo demorado es similar en pacientes con TCA y TDM siendo en ambos casos los rendimientos significativamente inferiores a los detectados en las personas sanas. Estos datos coinciden con lo constatado en estudios previos que comparan pacientes con TCA versus controles sanos (Ros-Cucurull et al., 2018; Stavro et al., 2013; Van Geldorp et al., 2012; Villa et al., 2021a; Villa et al., 2021b; Wester et al., 2014) y pacientes con TDM versus controles sanos

Tabla 3. Medias, medias ajustadas, desviaciones estándar y errores estándar para los diferentes índices del California Verbal Learning Test en los diferentes grupos en función del sexo.

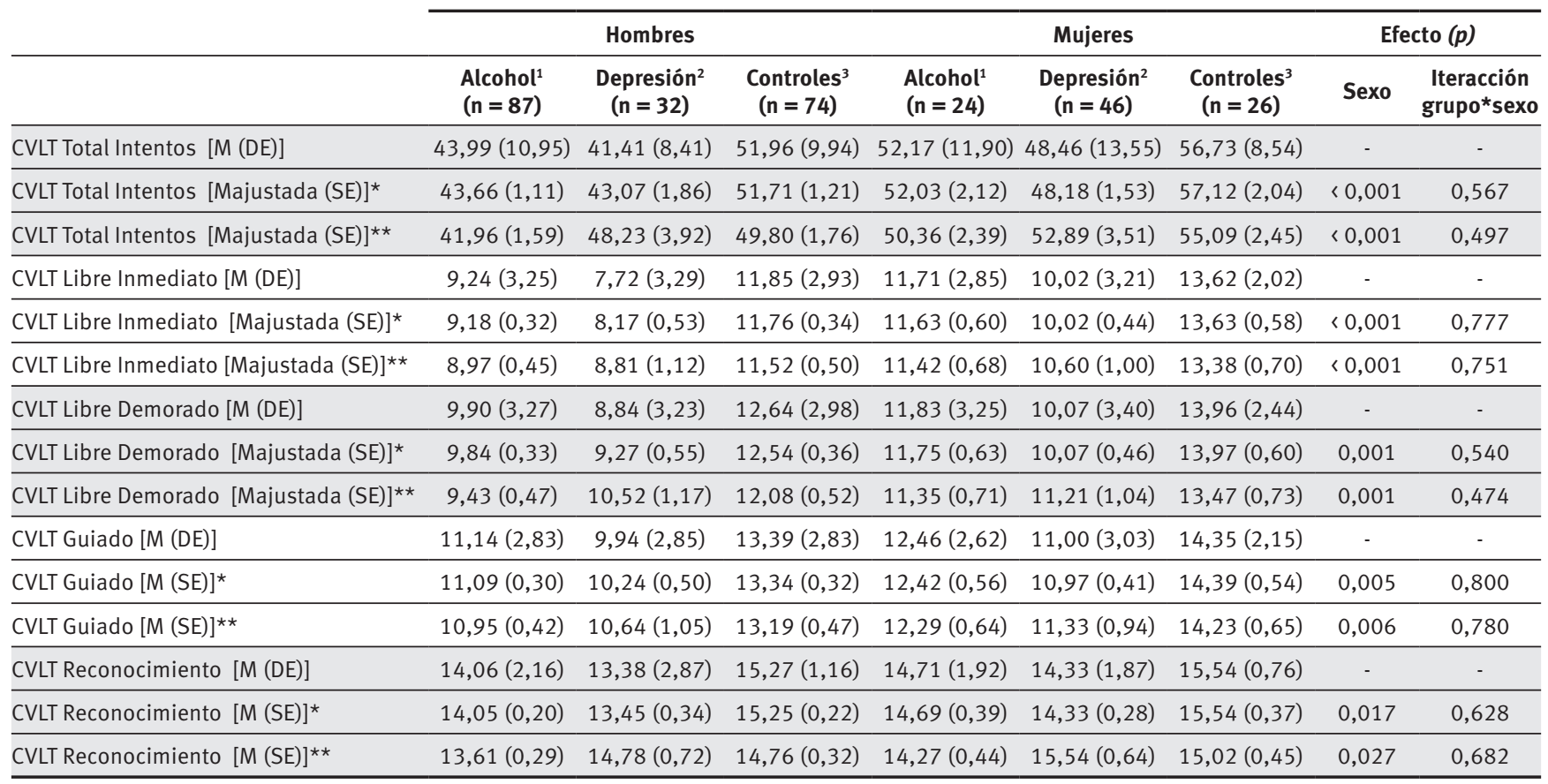

Nota. ${ }^{* *}$ Covariables: edad y años de estudios finalizados; ${ }^{* \star}$ Covariables: edad, años de estudios finalizados y puntuación en la Escala de Hamilton para la Depresión (HDRS); CVLT = California Verbal Learning Test; $M=$ media; $\mathrm{DE}=$ desviación estándar; $\mathrm{SE}=$ error estándar. 

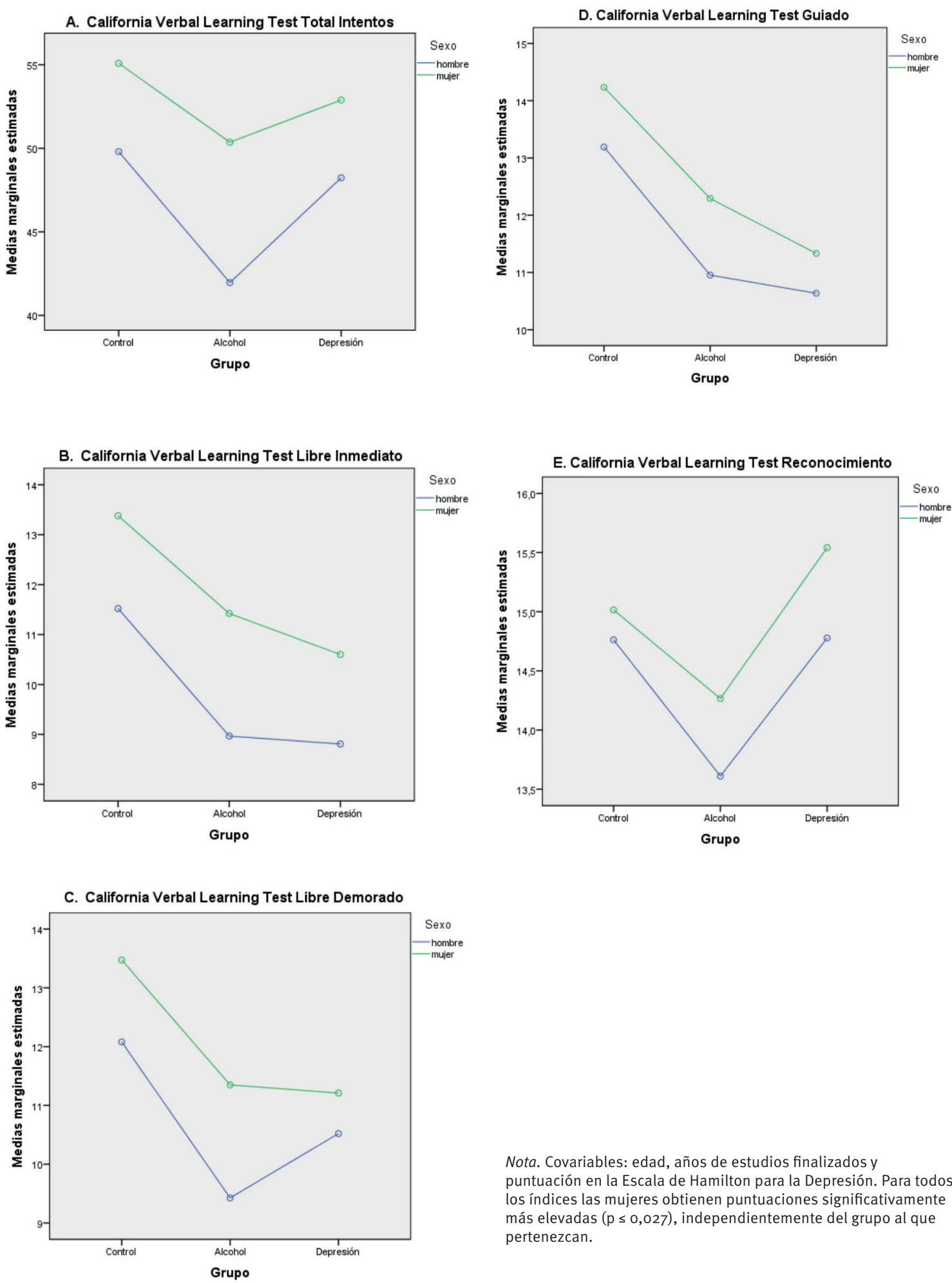

Nota. Covariables: edad, años de estudios finalizados y puntuación en la Escala de Hamilton para la Depresión. Para todos los índices las mujeres obtienen puntuaciones significativamente más elevadas $(p \leq 0,027)$, independientemente del grupo al que pertenezcan.

Figura 1. Medias ajustadas para los diferentes índices del California Verbal Learning Test en función del sexo y del grupo de estudio.

(Lee, Hermens, Porter y Redoblado-Hodge, 2012; Marazziti et al., 2010; Mesholam-Gately et al., 2012; Roca et al., 2015). Por otra parte, los pacientes con TDM obtienen peores puntuaciones en el índice de recuerdo libre guiado, es decir, en aquel en que se proporciona al paciente pistas categoriales para facilitar el recuerdo. Diversos autores defienden que los resultados obtenidos en el CVLT pueden estar mediados por el funcionamiento ejecutivo (Mesholam-Gately et al., 2012; Moreira, Santos, Sousa y Costa, 2015). Se podría hipotetizar que el recuerdo guiado con pistas categóricas precisaría de la intervención frontal para la asociación semántica favorecedora del recuerdo y esto podría influir en un peor rendimiento en pacientes con TDM, ya que ha sido descrita una importante altera- 
ción de la función ejecutiva en la depresión (Nuño, Gómez-Benito, Carmona y Pino, 2021; Rock et al., 2014). No obstante, cabe señalar que otros autores no encuentran diferencias significativas en el recuerdo guiado tardío entre pacientes con TDM y controles sanos (Fossatti, Deweer, Raoux y Allilaire, 1995).

Tras considerar los factores de confusión en el posterior análisis ANCOVA, se observa que los rendimientos en la memoria reciente y el reconocimiento continúan siendo similares y significativamente inferiores en pacientes con TCA y TDM en comparación con los controles sanos, con excepción de un peor rendimiento en el recuerdo libre inmediato y en el recuerdo guiado en los pacientes con TDM (versus TCA y controles). Datos previos evidencian que los pacientes deprimidos muestran dificultades con tareas de memoria verbal libre inmediata (Fossatti et al., 1995) existiendo un amplio acuerdo en que pueden ser secundarias a otras disfunciones, como el déficit atencional, la interferencia cognitiva de pensamientos rumiativos pesimistas, la falta de motivación, así como, la dificultad de mantener un esfuerzo sostenido (Marazzitti et al., 2010; Millan et al., 2012).

Se observa en este primer análisis que persiste una mayor dificultad de pacientes con TDM en el recuerdo guiado pudiendo inferirse que el recuerdo facilitado por pistas no se ve influido por los factores confusores tenidos en cuenta (edad, sexo y/o nivel de estudios completados).

Por otro lado, resulta interesante destacar que, en todos los grupos (TCA, TDM y controles sanos) y en todos índices de la prueba, las mujeres obtuvieron mejores rendimientos que los hombres. No se observó en ninguno de los índices, una interacción estadísticamente significativa entre sexo y grupo tras el correspondiente control por edad y años de educación completados. Estudios previos hacen referencia a que, en todos los rangos de edad, las mujeres superan a los hombres en aprendizaje y recuerdo verbal utilizando el CVLT, lo cual es consistente con la hipótesis que vincula el nivel de estrógenos y el rendimiento en la memoria verbal (Kramer et al., 2019; Lundervold, Wollschläger y Wehling, 2014).

Tras controlar por la gravedad de la depresión, sólo se detectan diferencias estadísticamente significativas entre el grupo de pacientes con TCA y los controles sanos. Por tanto, en nuestra muestra la gravedad de la depresión se erige como factor determinante de la afectación de la memoria verbal en los pacientes con TDM. Nuestros datos confirman estudios previos que encuentran que la gravedad de la depresión se asocia con un déficit en el aprendizaje y memoria verbal de los pacientes depresivos (Lee et al., 2015; Marazziti et al., 2010; McDermott y Ebmeier, 2009). Por otra parte, se confirma la hipótesis de trabajo inicial ya que los pacientes con TCA presentan un mayor grado de deterioro en todos los índices de la prueba CVLT al compararlos con pacientes con TDM y controles sanos. Estudios previos ponen de manifiesto que los pacientes con TCA que buscan tratamiento presentan claro deterioro cognitivo que incluye disfunción ejecutiva y déficits de memoria (Oscar-Berman et al., 2014; Stavro et al., 2013; Sullivan, Rosenbloom, Lim y Pfefferbaum, 2000). El consumo abusivo de alcohol daña el tejido cerebral provocando marcada atrofia cerebral generalizada, así como daño específico en las áreas encargadas del aprendizaje y la memoria (Ridley et al., 2013; Stavro et al., 2013; Zahr y Pfefferbaum, 2017). En pacientes con TCA se producen déficits en los procesos de codificación y recuperación verbal (Bernardin, Maheut-Bosser y Paille, 2014; Noël et al, 2001; Pitel et al., 2007) y podrían afectarse el aprendizaje de información verbal y no verbal (Kopera et al., 2012). Nuestros resultados confirman lo previamente descrito con respecto a la afectación de la memoria verbal en TCA (Ros-Cucurull et al., 2018; Van Geldorp et al., 2012; Villa et al., 2021a; Villa et al., 2021b; Wester et al., 2014). No obstante, cabe señalar, que los estudios realizados hasta la fecha comparan TCA con controles sanos no incluyendo otro grupo de pacientes que clásicamente se asocia con importante deterioro en la cognición como son los pacientes con TDM. Por tanto, nuestros resultados tienen una mayor relevancia tanto a nivel clínico como terapéutico. La memoria y el funcionamiento ejecutivo son dominios cognitivos estrechamente vinculados a los resultados del tratamiento y al mantenimiento de la abstinencia (Bates, Buckman y Nguyen, 2013). Esta disfunción en el aprendizaje y la memoria podría interferir en la adecuada asimilación de las intervenciones psicoterapéuticas, pudiendo disminuir la eficacia de las mismas, y dificultar la consecución del objetivo de abandono del hábito alcohólico (Bates et al., 2013; Florez et al., 2019; Sachdeva et al., 2016). Señalar que, si bien, existen estudios con datos que podrían resultar prometedores en la mejora de dicha disfunción cognitiva, utilizando abordajes tanto psicofarmacológicos (Bell, Pittman, Petrakis y Yoon, 2020; Sachdeva et al., 2016) como psicoterapéuticos (Frias-Torres et al., 2018; Hayes et al., 2016; Rupp, Kemmler, Kurz, Hinterhuber y Fleischhacker, 2012; Sachdeva et al., 2016; Svanberg et al., 2013), los resultados de estos trabajos no son concluyentes en la actualidad.

La principal limitación de este estudio es el diseño transversal del mismo que no permite evaluar el pronóstico de la disfunción de la memoria a medio/largo plazo. Otra limitación asociada al diseño transversal es la dificultad para medir retrospectivamente el consumo de alcohol con un mayor grado de rigor. Por otra parte, señalar que no se ha realizado determinación premórbida del cociente intelectual, que a su vez puede influir en el desempeño de la prueba. Señalar, por último, no se ha analizado la interferencia en la cognición de los tratamientos farmacológicos empleados. No obstante, también es necesario señalar la existencia de diversas fortalezas. La principal es que se 
trata del primer trabajo de estas características que evalúa el aprendizaje y memoria verbal en una muestra de pacientes con TCA en comparación con pacientes con TDM y controles sanos. De igual modo, la selección de la muestra ha sido muy estricta, excluyéndose pacientes con otras comorbilidades psiquiátricas, con el fin de poder establecer los factores específicos asociados a la memoria verbal en pacientes con TCA y TDM.

Señalar, por último, que, en nuestra muestra, se ha observado que los pacientes con TCA presentan una importante alteración en el aprendizaje y la memoria verbal al compararlos con pacientes con TDM y con personas sanas. Estos resultados plantean la necesidad de estudios futuros que analicen en qué medida este trastorno mnésico verbal puede influir, negativamente, en los resultados de un programa de deshabituación alcohólica. De modo que, podría ser conveniente adecuar las intervenciones terapéuticas al nivel cognitivo de cada paciente para favorecer la eficacia de las mismas.

\section{Agradecimientos}

Este estudio contó con el apoyo de la Delegación del Gobierno para el Plan Nacional sobre Drogas y de la Secretaría de Estado de Servicios Sociales e Igualdad del Ministerio de Sanidad y Consumo (Ref: 20161070), el Instituto de Salud Carlos III (FIS PI14/02029 y PI17/01433), el Ministerio Español de Economía y Competitividad, los Fondos Europeos de Desarrollo Regional (FEDER), el Gobierno del Principado de Asturias (PCTI 2018-2022 IDI/2018/235) y el Centro de Investigación Biomédica en Red de Salud Metal (CIBERSAM).

\section{Conflicto de intereses}

Los autores declaran la ausencia de conflictos de intereses en relación al estudio, su autoría, y / o a la publicación de este manuscrito.

\section{Referencias}

American Psychiatric Association. (2013). Diagnostic and Statistical Manual of Mental Disorders, 5th ed. (DSM-5). Arlington, VA: American Psychiatric Publishing.

Bates, M. E., Buckman, J. F. y Nguyen, T. T. (2013). A role for cognitive rehabilitation in increasing the effectiveness of treatment for alcohol use disorders. Neuropsychology Review, 23, 27-47. doi:10.1007/s11065-013-9228-3.

Bech, P. (1996). The Bech, Hamilton and Zung scales for mood disorders: Screening and listening, 2nd ed. Berlin: Springer.

Bell, M. D., Pittman, B., Petrakis, I. y Yoon, G. (2020). Donepezil and cognitive remediation therapy to augment treatment of alcohol use disorder related mild cognitive impairment (AUD-MCI): An open label pilot study with historical controls. Substance abuse. Avance de publicación on-line. doi:10.1080/08897077.2020.1844847.

Bernardin, F., Maheut-Bosser, A. y Paille, F. (2014). Cognitive impairments in alcohol-dependent subjects. Frontiers in Psychiatry, 16, 78. doi:10.3389/fpsyt.2014.00078.

Bobes, J., Bulbena, A., Luque, A., Dal-Re, R., Ballesteros, J. e Ibarra, N. (2003). Grupo de validación en español de escalas psicométricas. Evaluación psicométrica comparativa de las versiones en español de 6, 7 y 21 ítems de la Escala de valoración de Hamilton para la evaluación de la depresión. Medicina Clínica, 120, 693-700.

Bortolato, B., Miskowiak, K. W., Köler, C. A., Maes, M., Fernandes, B. S., Berk, M. y Carvalho, A. F. (2016). Cognitive remission: A novel objective for the treatment of major depression? BMC Medicine, 14, 9. doi:10.1186/ s12916-016-0560-3.

Brust, J. C. M. (2010). Ethanol and cognition: Indirect effects, neurotoxicity and europrotection: A review. International Journal of Environmental Research and Public Health, 7, 1540-1557. doi:10.3390/ijerph7041540.

Crowe, S. F., Cammisuli, D. M. y Stranks, E. K. (2019). Widespread cognitive deficits in alcoholism persistent following prolonged abstinence: An updated meta-analysis of studies that used standardised europsychological assessment tools. Archives of Clinical Neuropsychology, 35, 31-45. doi:10.1093/arclin/acy106.

Delis, D. C., Kramer, J. H., Kaplan, E. y Ober, B. A. (1987). California Verbal Learning Test. Research edition manual. New York: Psychological Corporation.

Erdozain, A. M., Morentin, B., Bedford, L., King, E., Tooth, D., Brewer, C.,... Carter, W. G. (2014). Alcohol-related brain damage in humans. PloS One, 9. doi:10.1371/journal.pone.0093586.

Florez, G., Espandian, A., Villa, R. y Saiz, P. A. (2019). Clinical implications of cognitive impairment and alcohol dependence. Adicciones, 31, 3-7. doi:10.20882/adicciones.1284.

Fossatti, P., Deweer, B., Raoux, N. y Allilaire, J. F. (1995). Deficits in memory retrieval: An argument in favor of frontal subcortical dysfunction in depression. L'Encephale, 21, 295-305.

Fossatti, P., Coyette, F., Ergis, A. M. y Allilaire, J. F. (2002). Influence of age and executive functioning on verbal memory of inpatients with depression. Journal of Affective Disorders, 68, 261-271. doi:10.1016/s01650327(00)00362-1.

Frias-Torres, C., Moreno-Espana, J., Ortega, L., Barrio, P., Gual, A. y Teixidor Lopez, L. (2018). Remediation therapy in patients with alcohol use disorders and neurocognitive disorders: A pilot study. Adicciones, 30, 93-100. doi:10.20882/adicciones.757.

Gregory, E., Torres, I. J., Ge, R., Blumberger, D. M., Downar, J. H., Daskalakis, Z. J.,... Vila-Rodriguez, F. (2020). Predictors of cognitive impairment in treatment-resis- 
tant depression. Journal of Affective Disorders, 274, 593601. doi:10.1016/j.jad.2020.05.10.

Hamilton, M. (1960). A rating scale for depression. Journal of Neurology Neurosurgery and Psychiatry, 23, 56-62. doi:10.1136/jnnp.23.1.56.

Hayes, V., Demirkol, A., Ridley, N., Withall, A. y Draper, B. (2016). Alcohol-related cognitive impairment: Current trends and future perspectives. Neurodegenerative Disease Management, 6, 509-523. doi:10.2217/nmt-2016-0030.

Hunt, S. A., Baker, A. L., Michie, P. T. y Kavanagh, D. J. (2009). Neurocognitive profiles of people with comorbid depression and alcohol use: Implications for psychological interventions. Addictive behaviors, 34, 878-886. doi:10.1016/j.addbeh.2009.03.036.

Hunt, S. A., Kay-Lambkin, F. J., Baker, A. L. y Michie, P. T. (2015). Systematic review of neurocognition in people with co-occurring alcohol misuse and depression. Journal of affective disorders, 179, 51-64. doi:10.1016/j. jad.2015.03.024.

Kopera, M., Wojnar, M., Brower, K., Glass, J., Nowosad, I., Gmaj, B. y Szelenberger, W. (2012). Cognitive functions in abstinent alcohol-dependent patients. Alcohol, 46, 666-671. doi:10.1016/j.alcohol.2012.04.005.

Kramer, A. O., Casaletto, K. B., Umlauf, A., Staffaroni, A. M., Fox, E., You, M. y Kramer, J. H. (2019). Robust normative standards for the California Verbal Learning Test (CVLT) ages 60-89: A tool for early detection of memory impairment. Clinical Neuropsychologist, 34, 384-405. doi:10.1080/13854046.2019.1619838.

Kuźma, E., Llewellyn, D. J., Langa, K. M., Wallace, R. B. y Lang, I. A. (2014). History of alcohol use disorders and risk of severe cognitive impairment: A 19-year prospective cohort study. American Journal of the Geriatric Psychiatry, 22, 1047-1054. doi:10.1016/j.jagp.2014.06.001.

Le Berre, A. P., Fama, R. y Sullivan, E. V. (2017). Executive functions, memory, and social cognitive deficits and recovery in chronic alcoholism: A critical review to inform future research. Alcoholism, Clinical and Experimental Research, 41, 1432-1443. doi:10.1111/acer.13431.

Lee, R. S., Dore, G., Juckes, L., De Regt, T., Naismith, S. L., Lagopoulos, J.,... Hermens, D. F. (2015). Cognitive dysfunction and functional disability in alcohol-dependent adults with or without a comorbid affective disorder. Cognitive neuropsychiatry, 20, 222-231. doi:10.1080/1354 6805.2015.1014031.

Lee, R. S., Hermens, D. F., Porter, M. A. y Redoblado-Hodge, M. A. (2012). A meta-analysis of cognitive deficits in first-episode major depressive disorder. Journal of Affective Disorders, 140, 113-124. doi:10.1016/j.jad.2011.10.023.

Lundervold, A. J., Wollschläger, D. y Wehling, E. (2014). Age and sex related changes in episodic memory function in middle aged and older adults. Scandinavian Journal of Psychology, 55, 225-232. doi:10.1111/sjop.12114.
Maharasingam, M., Macniven, J. A. y Mason, O. J. (2013). Executive functioning in chronic alcoholism and Korsakoff syndrome. Journal of Clinical and Experimental Neuropsychology, 35, 501-508. doi:10.1080/13803395.2013.79 5527.

Marazziti, D., Consoli, G., Picchetti, M., Carlini, M. y Faravelli, L. (2010). Cognitive impairment in major depression. European Journal of Pharmacology, 626, 83-86. doi:10.1016/j.ejphar.2009.08.046.

McDermott, L. M. y Ebmeier, K. P. (2009). A meta-analysis of depression severity and cognitive function. Journal of Affective Disorders, 119, 1-8. doi:10.1016/j. jad.2009.04.022.

Mesholam-Gately, R. I., Giuliano, A. J., Zillmer, E. A., Barakat, L. P., Kumar, A., Gur, R. C.,... Moberg, P. J. (2012). Verbal learning and memory in older adults with minor and major depression. Archives of Clinical Neuropsychology, 27, 196-207. doi:10.1093/arclin/acr106.

Millan, M. J., Agid, Y., Brüne, M., Bullmore, E. T., Carter, C. S., Clayton, N. S.,... Young, L. J. (2012). Cognitive dysfunction in psychiatric disorders: Characteristics, causes and the quest for improved therapy. Nature Reviews Drug Discovery, 11, 141-168. doi:10.1038/nrd3628.

Moreira, P. S., Santos, N. C., Sousa, N. y Costa, P. S. (2015). The use of canonical correlation analysis to assess the relationship between executive functioning and verbal memory in older adults. Gerontology and Geriatric Medicine, 1. doi:10.1177/2333721415602820.

Moretti, R., Caruso, P., Dal Ben, M., Gazzin, S. y Tiribelli, C. (2017). Thiamine and alcohol for brain pathology: Super-imposing or different causative factors for brain damage? Current Drug Abuse Reviews, 10, 44-51. doi:10.21 74/1874473711666180402142012.

Noël, X., Van der Linden, M., Schmidt, N., Sferrazza, R., Hanak, C., Le Bon, O.,... Verbanck, P. (2001). Supervisory attentional system in nonamnesic alcoholic men. Archives of General Psychiatry, 58, 1152. doi:10.1001/archpsyc.58.12.1152.

Nuño, L., Gómez-Benito, J., Carmona, V. R. y Pino, O. (2021). A systematic review of executive function and information processing speed in major depression disorder. Brain Sciences, 11, 147. doi:10.3390/brainsci11020147.

Oscar-Berman, M., Valmas, M. M., Sawyer, K. S., Ruiz, S. M., Luhar, R. B. y Gravitz, Z. R. (2014). Profiles of impaired, spared, and recovered neuropsychological processes in alcoholism. Handbook of Clinical Neurology, 125, 183-210. doi:10.1016/B978-0-444-62619-6.00012-4.

Pitel, A. L., Beaunieux, H., Witkowski, T., Vabret, F., Guillery-Girard, B., Quinette, P.,... Eustache, F. (2007). Genuine episodic memory deficits and executive dysfunctions in alcoholic subjects early in abstinence. Alcoholism, Clinical and Experimental Research, 31, 1169-1178. doi:10.1111/j.1530-0277.2007.00418.x. 
Ridley, N. J., Draper, B. y Withall, A. (2013). Alcohol-related dementia: An update of the evidence. Alzheimer's Research and Therapy, 5, 3. doi:10.1186/alzrt157.

Rock, P. L., Roiser, J. P., Riedel, W. J. y Blackwell, A. D. (2014). Cognitive impairment in depression: A systematic review and meta-analysis. Psychological Medicine, 44, 2029-2040. doi:10.1017/S0033291713002535.

Roca, M., Vives, M., López-Navarro, E., García-Campayo, J. y Gili, M. (2015). Cognitive impairments and depression: A critical review. Actas Españolas de Psiquiatría, 43, 187-193.

Ros-Cucurull, E., Palma-Alvarez, R. F., Cardona-Rubira, C., Garcia-Raboso, E., Jacas, C., Grau-Lopez, L.,... Roncero, C. (2018). Alcohol use disorder and cognitive impairment in old age patients: A 6 months follow-up study in an outpatient unit in Barcelona. Psychiatry Research, 261, 361-366. doi:10.1016/j.psychres.2017.12.069.

Rupp, C. I., Kemmler, G., Kurz, M., Hinterhuber, H. y Fleischhacker, W. W. (2012). Cognitive remediation therapy during treatment for alcohol dependence. Journal of studies on alcohol and drugs, 73, 625-634. doi:10.15288/ jsad.2012.73.625.

Sachdeva, A., Chandra, M., Choudhary, M., Dayal, P. y Anand, K. S. (2016). Alcohol-related dementia and neurocognitive impairment: A review study. International Journal of High Risk Behaviors and Addiction, 5. doi:10.5812/ijhrba.27976.

Spear, L. P. (2018). Effects of adolescent alcohol consumption on the brain and behaviour. Nature Reviews Neuroscience, 19, 197-214. doi:10.1038/nrn.2018.10.

Stavro, K., Pelletier, J. y Potvin, S. (2013). Widespread and sustained cognitive deficits in alcoholism: A meta-analysis. Addiction Biology, 18, 203-213. doi:10.1111/j.13691600.2011.00418.x.

Sullivan, E. V., Rosenbloom, M. J., Lim, K. O. y Pfefferbaum, A. (2000). Longitudinal changes in cognition, gait, and balance in abstinent and relapsed alcoholic men: Relationships to changes in brain structure. Neuropsychology, 14, 178-188.

Svanberg, J. y Evans, J. J. (2013) . Neuropsychological rehabilitation in alcohol-related brain damage: A systematic review. Alcohol and Alcoholism, 48, 704-711. doi:10.1093/ alcalc/agt131.

Toledo-Nunes, P., Kipp, T. K., Reitz, N. L. y Savage, L. M. (2019). Aging with alcohol-related brain damage: Critical brain circuits associated with cognitive dysfunction. International Review of Neurobiology, 148, 101-168. doi:10.1016/bs.irn.2019.09.002.

Topiwala, A., Allan, C. L., Valkanova, V., Zsoldos, E., Filippini, N., Sexton, C.,... Ebmeier, K. P. (2017). Moderate alcohol consumption as risk factor for adverse brain outcomes and cognitive decline: Longitudinal cohort study. BMJ, 357. doi:10.1136/bmj.j2353.
Van Geldorp, B., Bergman, H. C., Robertson, J., Wester, A. J. y Kessels, R. P. C. (2012). The interaction of working memory performance and episodic memory formation in patients with Korsakoff's amnesia. Brain Research, 1433, 98-103. doi:10.1016/j.brainres.2011.11.036.

Villa, R., Espandian, A., Saiz, P. A., Astals, M., Valencia, J. K., Martinez-Santamaria, E.,... Florez G. (2021a). Cognitive functioning in patients with alcohol use disorder who start outpatient treatment. Adicciones, 33, 161-174. doi:10.20882/adicciones.1326.

Villa, R., Espandian, A., Saiz, Rodriguez-Revuelta, J., Garcia-Portilla, P., Bobes, J. y Florez, G. (2021b). Cognitive functioning after six months of follow-up in a sample of alcohol use disorder outpatients. Adicciones. Avance de publicación on-line. doi:10.20882/adicciones.1672.

Wester, A. J., Roelofs, R. L., Egger, J. I. M. y Kessels, R. P. C. (2014). Assessment of alcohol-related memory deficits: A comparison between the Rivermead Behavioural Memory Test and the California Verbal Learning Test. Brain Impairment, 15, 18-27. doi:10.1017/BrImp.2014.6.

Wollenweber, F. A., Halfter, S., Brugmann, E., Weinberg, C., Cieslik, E. C., Muller, V. I.,... Eickhoff, S. B. (2014). Subtle cognitive deficits in severe alcohol addicts-do they show a specific profile? Journal of Neuropsychology, 8 , 147-153. doi:10.1111/jnp.12001.

World Medical Association. (2013). World Medical Association Declaration of Helsinki: Ethical principles for medical research involving human subjects. JAMA, 310, 2191-2194. doi:10.1001/jama.2013.281053.

Zahr, N. M. y Pfefferbaum, A. (2017). Alcohol's effects on the brain: Neuroimaging results in humans and animal models. Alcohol Research: Current Reviews, 38, 183-206. 